\title{
Determinants of Nutritional Condition: Discordance between Existing Understanding and Current Observation- A Study among the School Students of West Bengal
}

\author{
Tapas Saha ${ }^{1}$, Tushar Kanti Pathak ${ }^{2}$, Partha Sarathi Mukherjee ${ }^{1, *}$ \\ ${ }^{1}$ Liver Foundation, West Bengal, 12 Kyd Street, Kolkata, India \\ ${ }^{2}$ Department of Health and Family Welfare, Government of West Bengal, India \\ *Corresponding author: spartham@gmail.com
}

Received August 15, 2014; Revised August 27, 2014; Accepted September 06, 2014

\begin{abstract}
Ensuring food security and nutrition in India is a great challenge considering continuous increase in population. Every adult individual should understand nutrition and the reason of malnutrition (under and over). For students, understanding of proper nutrition is a responsibility of their parents and teachers primarily. As food is the fuel of human living and correct food supplement is the key of growth and development, students should be guided accordingly to secure their future and it's more important when few of them will dream to be successful in sports. In general participation in sports and games should be for all, every student needs to do regular exercise and thus understanding of nutrition is essential. Therefore, to identify the determinants of nutritional condition in a population, particularly in student community will have some significance in health education and can be useful also in sports science. This study is aimed to review the determinants of nutritional condition of the school students for example, parental education, occupation and number of family members on their child nourishment and to identify the social basis of growth and development in children and adolescent. The data were collected by Sarbashiksha Mission, Bardhaman; Government of West Bengal. To define underweight and obese category, we used the sex- and agespecific body mass index cutoffs as referred by Khadilkar et al (Indian Pediatrics). Parental socioeconomic determinants have not had any significant effect always on their child's nourishment. This study reveals that existing understanding about the parental education, occupation, number of family members and following impact on the nutrition of their children is not correct. Financially affluent people will have more fat, while poor people are suffering with under nutrition, higher education can influence proper growth and more members in a family will contribute a lesser amount of food distributed to each individual if the family is not well-off, these are the existing understanding with regard to nutrition. But there is a discordance between existing understanding and current observation. It is revealed in this study that we need to understand nutrition in a holistic approach rather than driven by conventional thinking. It is observed, parents, teacher must be aware that socioeconomic conditions are not the only determinants of nutritional condition, perhaps we need to have the proper health education, knowledge of correct food supplement can ensure proper nutrition of children and adolescent even in underprivileged section. We have to find out the probable reasons for such scenario, students are the future of this country, they should be guided by a correct understanding of nutrition, the role of exercise in daily life to ensure the proper nutrition.
\end{abstract}

Keywords: education, occupation, underweight, obesity, students, exercise, children

Cite This Article: Tapas Saha, Tushar Kanti Pathak, and Partha Sarathi Mukherjee, "Determinants of Nutritional Condition: Discordance between Existing Understanding and Current Observation- A Study among the School Students of West Bengal.” American Journal of Sports Science and Medicine, vol. 2, no. 5A (2014): 23-28. doi: 10.12691/ajssm-2-5A-6.

\section{Introduction}

Though nutrients are the building blocks and on the basis of these a proper body structure will develop, but there is a very close relationship between nutrition and exercise to maintain a healthy weight. Consistent exercise and proper nutrition work synergistically, creating the mental ability for enhanced performance. But Several studies have shown that adoloscents' intake of important nutrients as well as their performance on the standard physical fitness test, has fallen in recent years. Nutrition is a much discussed issue across the world in the present time, both for the persistent problems of under nutrition and a growing problem of obesity and associated diseases, but remain a neglected area globally. India ranks first with $39 \%$ of the global share for underweight children with 
$47 \%$ of prevalence. [2] Today, 27\% of children in the developing countries are underweight - that is about 146 million children. [3] The prevalence of underweight children in India is highest in the world. Child malnutrition in School going children is responsible for $22 \%$ of the country's burden of diseases. [4] More importantly, it has been seen that $16.9 \%$ of West Bengal (rural) children aged between 6-14 years are underweight. Under-nutrition is a process whose consequences have an impact not only in later life, but also into future generations. Deficiencies of key vitamins and minerals continue to be pervasive and they overlap considerably with the problem of general under nutrition. [5] In growing children, underweight or obesity affects intelligence and physical capacity. As a result, reduces productivity, slows economic growth and aggravates poverty. The cost incurred on malnutrition is very high. [6] In the developing countries, particularly in low- and middle-income families, child under- nutrition is still a primary concern for its direct effects on morbidity, mortality and human capital, as well as its link to chronic diseases in adulthood, particularly in countries undergoing nutritional transition. Simultaneously, the unabated rise in the prevalence of obesity in children and adolescents is one of the most alarming public health issues facing the world today. [7] Among Indian children, various studies report the magnitude of obesity from 1 to $12.9 \%$. [8] Obesity increases the risk for many chronic diseases, including diabetes mellitus, cardiovascular disease, and non-alcoholic fatty liver disease, and decreases the overall quality of life. Therefore, it is imperative to identify the 'at risk' individuals at an early stage. [7]

This paper addresses three key questions. First, does the education of parents, and particularly that of mothers, have a significant role in increasing the nutritional status of children? Child health is one of the main commodities produced within the household, and in essence, is the responsibility of mothers. Consequently, the human capital embodied in a mother can have a significant impact on a child's health status. [9] More education results in greater knowledge and better access to and processing of information (Thomas, Strauss and Henrique's 1990). In the context that better educated parents are more successful in protecting or improving their children's health status, public health programs intended at reducing child under nutrition and obesity. Identifying the significant connection between child health and education of parents is effective to construct a better health policy [9].

Second, does the occupation of parents have a significant role in increasing the nutritional status of children? Socio-demographic determinants are one of the most widely studied constructs in the social sciences. The three main parameters of socio-demographic status are, number of family members, parental education, and occupational status. Research indicates that sociodemographic determinants are linked to a wide array of child nutrition, cognitive development, and socioemotional outcomes in children, with effects beginning prior to birth and continuing into adulthood. Its effects are endured by children's own characteristics, household characteristics and by developing surrounding environmental scenario. [10] It is well established that "The problems of over- and under nutrition are not simply a problem of rich or poor, respectively,"."To the contrary, all too often these problems overlap and coexist. Currently, strategies to tackle this dual burden of malnutrition are often pursued and promoted independently, as if the two problems were independent of each other. This will need to change, and policies and programmes developed that address both issues in an integrated manner." [11]

The third question that naturally arises, is there any impact of family members on child nourishment? Or, does the allocation of foods to family members when the number of members in a family is considerably high, play a pivotal role in relation to the nutritional health of children and adolescents? It is mainly observed in the rural sector where the illumination of knowledge could not reach properly and the people have to struggle relentlessly against poverty to collect their everyday meal. It is well known and very common concept for the Indians that sharing of foods in a family member may act as a protective factor for many nutritional health-related problems during childhood and adolescence, including issues of underweight, overweight, unhealthy eating. These inconsistencies may stem from the variability in ages examined and the concentration on both sexes rather than examining them one by one. [13] In this study, we aimed to ascertain the effectiveness of the relationship between the number of household members and children's nutritional health.

From the above discussion, it is noted that several factors affect school going children's health. Still more work is needed to identify the further influential factors, which can improve the nutritional status among children in rural areas of Bardhaman district. In our present study for the purpose of analysis, we employ body mass index (BMI) as a simple and reliable measure of nutritional status besides allowing for the ease of measuring height and weight in the field setting. Body Mass Index (BMI) is believed to be an acceptable indicator of the risk of underweight and overweight in children and adolescents. It is expected that the findings will lead to consider alternative program strategies for the reduction of poor nutritional status as well as the problem of obesity among the children aged between 5-16 years.

Proper nutrition and physical activity play a vital role to maintain overall health and quality of life and nutritional status can play a large part in ones athletic performance. This study aims to measure the nutritional condition of the students of West Bengal and attempts to brings out the plausible reason of discordance between pre existing understanding and current observation. School going children are the basis of better future of any country and their nutritional needs are essential for the well-being of society. The age of 5 to 16 years is a period of transition between childhood and adulthood, which occupies an important phase in the life of mankind. This phase is characterised by an extremely rapid rate of growth [4].

School is the easiest way to reach large portions of the school age population. [4,13] In order to tackle the malnutrition there is a dire need to address this issue with sufficient awareness and relook at the nutritional scenario of children with regard to the prevalence of underweight and obesity. In our present study, we observe whether there is any impact of parental education, number of family members and type parental occupation on children's health and nutritional status. Therefore, the 
present study was undertaken with an objective to assess the nutritional status of rural School going children in terms of prevalence and severity of malnutrition (underweight and obesity) and to get the trend of underweight and obesity among rural school children aged between 5 to 16 years.

\section{Materials and Methods}

The present study was conducted both in panchayet and municipal area of Bardhaman district, West Bengal. As per 2011 census, out of the total population in the district, 39.89 percent lives in urban regions and 60.11 percent lives in rural areas of the villages. We generally focus on rural population wherein the list of schools was selected from a number of schools. After all set up, the information on the socio-demographic profile of the subjects was collected. Information regarding name, age, sex, class, parents' education and occupation, number of brothers and sisters (excluding the students) was collected. Personnel specifically trained for this purpose recorded the anthropometric measurements. Height was measured by the anthropometric rod and weight measurement was made by standard digital weighing scale. Both the equipments were standardized at regular intervals. Based on height and weight we calculate body mass index (BMI) for each child using the formula BMI = Weight $(\mathrm{kg}) /$ [Height (m) x Height (m)]. BMI is the best predictor for child nutritional status. Hence, in this present study, we computed age specific BMI values for the children aged between 5 to 16 years and based on this, we classified whether a child is underweight, normal weight, overweight and obese. The age specific cutoff values for BMI were developed from the research paper of Indian Pediatrics.

\subsection{Statistical Analysis}

After the collection of data, analysis of the same was performed. The process of data cleaning and appropriate representation was conducted for better analytical purposes. Duplicate values were deleted and statistical methods like't-test' were performed. Additionally, mean values were calculated as and when required.

Exploratory data analysis has also been made based on BMI values for the underweight and obesity; percentage of the same is computed based on three factors, which are - parental education, parental occupation and number of family members.

\section{Results}

The number of students participated in this study was 15357 among which 7651 Boys and 7706 Girls.

1. The following pie chart depicts the ratio of male and female children.

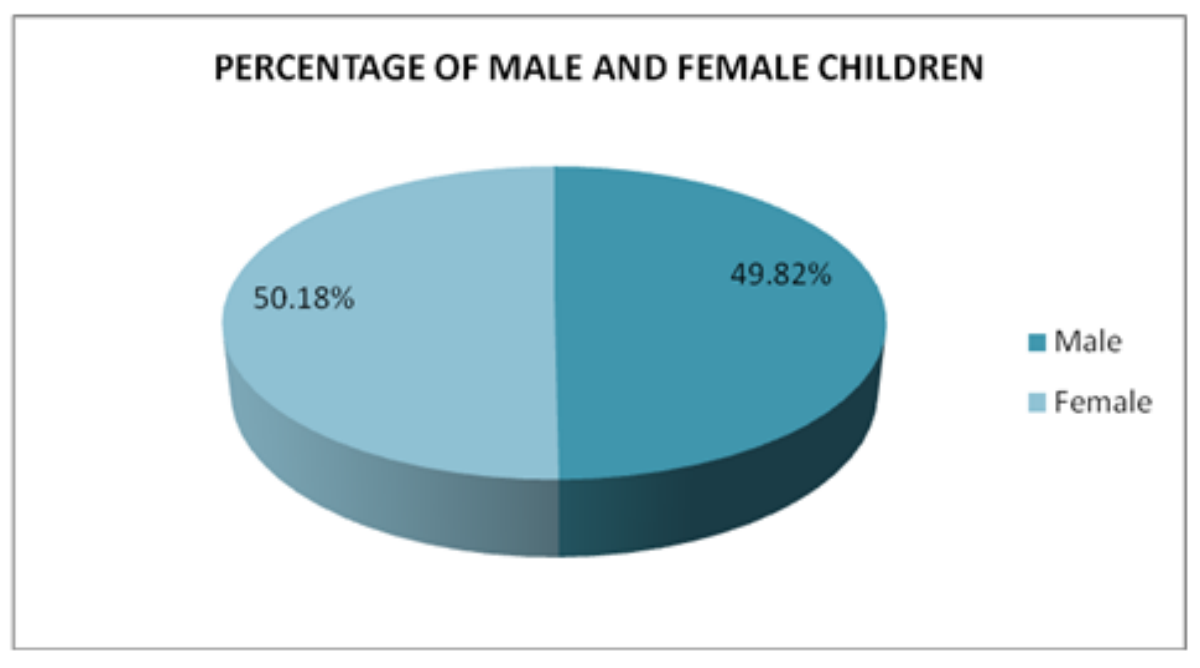

Figure 1. Pie Chart Showing the ratio of male and female children

From the above pie chart we see that there are approximately equal number of male and female children and since the data was collected randomly, at this point we can say that child sex ratio in the district of Bardhaman is exceedingly good.

2. The following table shows the socio-economic condition of rural sector of Burdwan District.

From the above table it is seen that the overall level of parental educational qualification is abysmally low, and the picture is the same, regardless of parents having a male or a female child. The picture becomes even darker when the fact comes to light that only $0.69 \%$ of the parents having a male child and $0.70 \%$ of the parents having a female child are either undergraduate or graduate. Obviously there are many factors affecting their education, but we need to identify those factors with proper manner and diligence. This is observed from this table that the percentage of families, those have three children are very high both in case of male and female child (41.24\% and $39.55 \%)$. The data also reveal that $48.22 \%$ are day labours and $21.05 \%$ are farmers in the said district clearly depicting the primary source of income.

Impact of Parental Education on Underweight and Obesity:

From this exhaustive study, it has been established that the relationship between parental education and child health is not very significant. The following column diagrams will exhibit the relationship between parental education and corresponding percentage of underweight and obese children. 


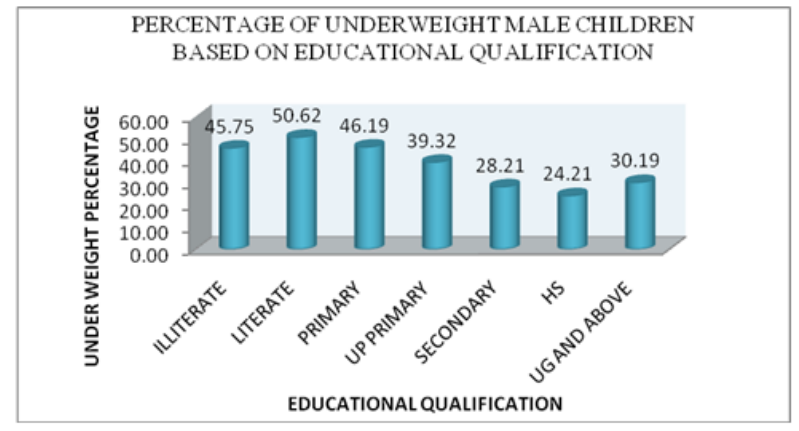

Figure 2. Column diagram shows the trend of underweight percentages for Male children

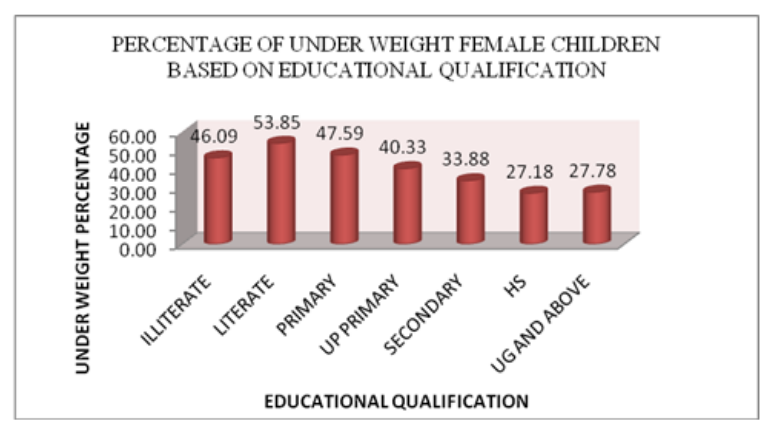

Figure 3. Column diagram shows the trend of underweight percentages for Female children

The column diagrams above show that there is not very much variation of percentages on the basis of educational qualification. We are generally acquainted with the knowledge that educational qualification and health are proportionally related, but our study shows that although the underweight percentage reduces from literate to higher secondary in both the diagrams, it again rises after HS.But on the overall we can say that the higher education of parents leads to drop in underweight percentage of the children.

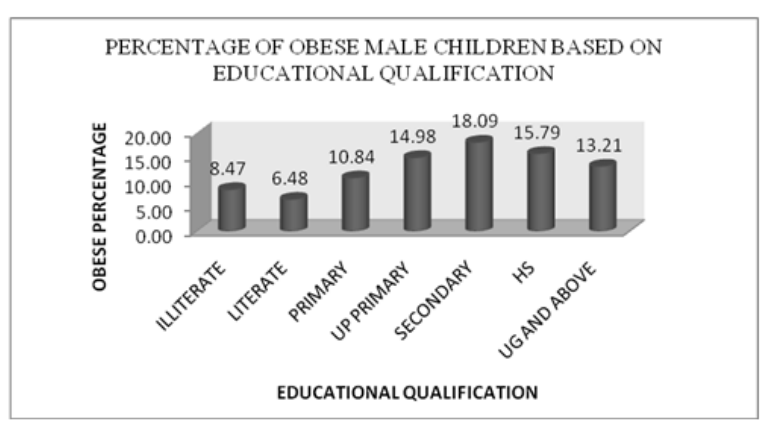

Figure 4. Column diagram shows the trend of obesity percentages for Male children

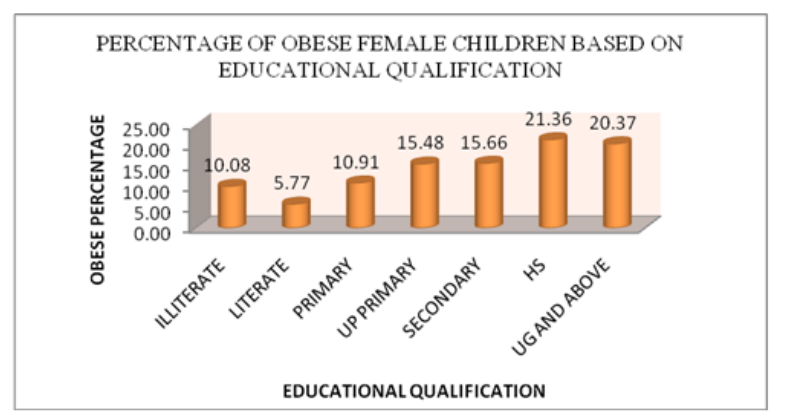

Figure 5. Column diagram shows the trend of obesity percentages for Female children
In contrast to Figure 2 and Figure 3, the above two diagrams exhibit relatively the same pattern,showing that advancement of educational qualification from literate to higher secondary leads to the steady increase in obesity percentage and for the under-graduates and above, there is a downward trend with respect to higher secondary. That is obesity increases, according to the level of educational qualification increases. Thus, from the above figure we conclude that irrespective of illiterate and literate, higher education of parents leads to obesity of their children. It is to be noted that in most of the above cases $(p<0.05)$ educational qualification is not a significant factor.

Impact of Family Members on Under Weight and Obesity:

The food security is always a big challenge in the densely populated country like India. Malnutrition, particularly under nutrition is supposed to be higher with the increase of family members in lower and middle income group. But surprisingly, it is not observed in the present study and the following two diagrams demonstrate how the underweight and obesity percentages change among Male and Female school children with the increase in the number of family members.

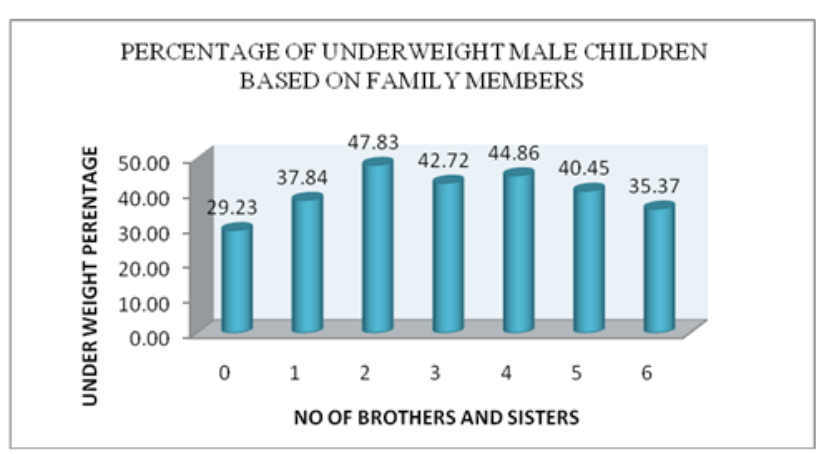

Figure 6. Column diagram shows the trend of underweight percentages for Male children

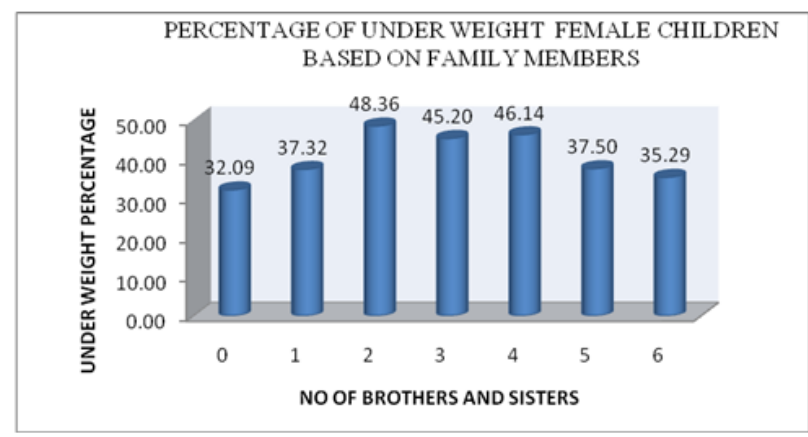

Figure 7. Column diagram shows the trend of underweight percentages for Female children

Note: On each of the above two graphs, in the $\mathrm{X}$-axis 0 denotes there is one child in a family, 1 denotes two children in a family and so on.

This is revealed that persentage of under weight remains high in both male and female students as well it is identified that the maximum under nutrition observed in families with three children in both casese. From the above figures, we find out that no significant difference $(p<0.05)$ in percentage for increasing the number of members in a family. 
Now we take a look into what happens in case of obesity and to illustrate this, the following diagrams are given.

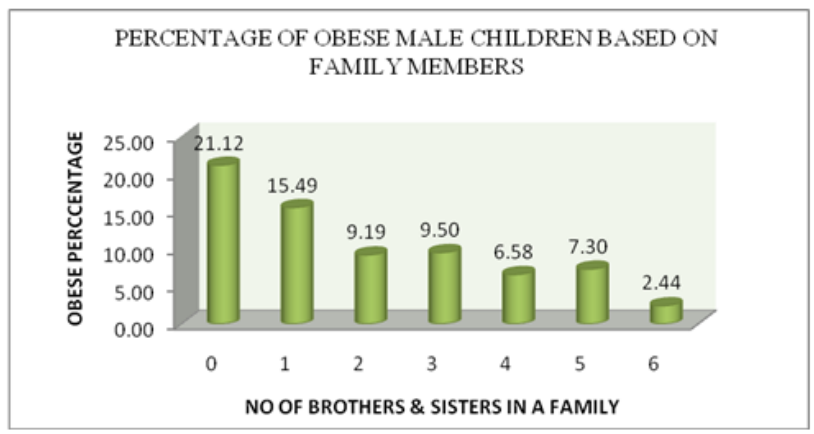

Figure 8. Column diagram shows the trend of obesity percentages for Male children

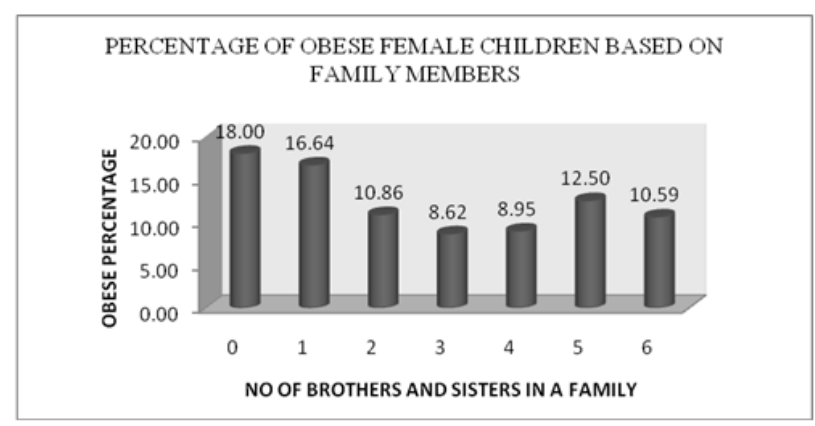

Figure 9. Column diagram shows the trend of obesity percentages for Female children

Note: On the above two graphs, in $\mathrm{X}$-axis 0 denotes there is one child in a family, 1 denotes two children in a family and so on.

From the above two scenarios we can explain that obesity is higher in those classes with one or two children in both male and female students and decrease with the increase of family members in both cases.

Impact of Parental Occupation on Under Weight and Obesity:

It is well established that parental income is positively associated with virtually every dimension of child wellbeing that social scientists measure. [14] Parents play the most important role in child growth; therefore their occupation in terms of income is very crucial. In particular, fathers' and mothers' occupation affects both the income coming into the family and the time devoted to children's development. [14] This study aimed to measure the effects of parent's occupation (type of job) on their child nutrition.

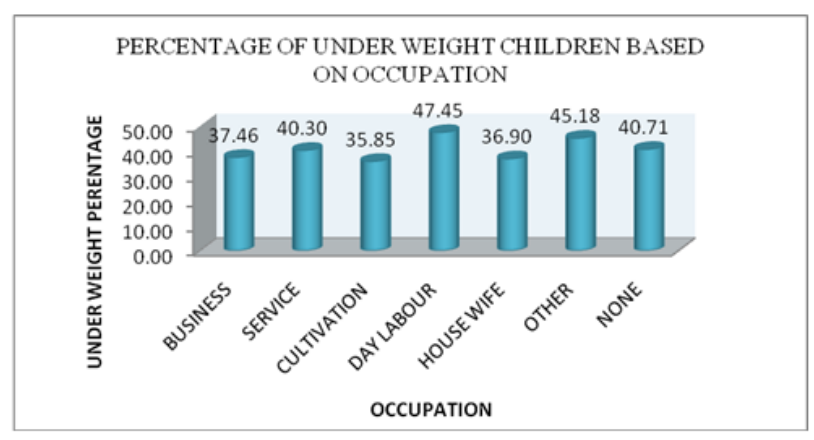

Figure 10. Column diagram shows the trend of underweight percentages for children
The above chart portrays that the trend of underweight percentage is very high irrespective of the parents' occupation. It is also noted that there is no significant difference among the underweight percentages of children with respect to the parents' occupation.

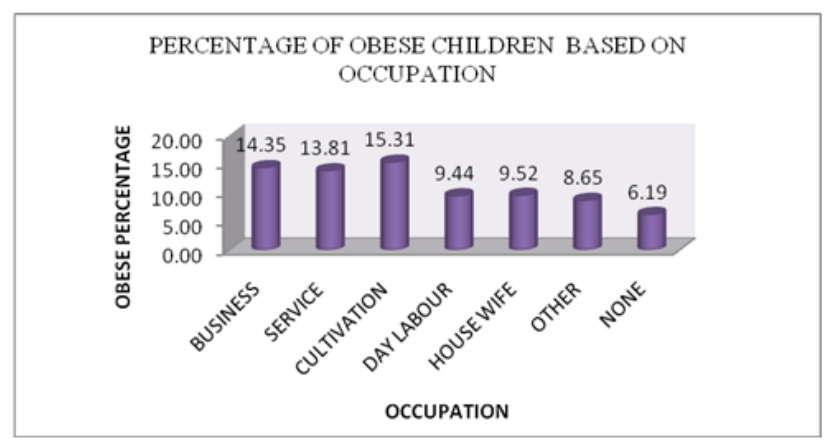

Figure 11. Column diagram shows the trend of obesity percentages for children

Again, we can see from the above diagram that there is no such significant difference in the percentage of obesity $(\mathrm{p}<0.05)$ with respect to type of occupation.

\section{Discussion}

This study reflects distinctive comparative information on trends in childhood and adolescents' underweight and obese status of Bardhaman district, West Bengal. From the review of our study, we can conclude that the prevalence of underweight and obesity among school going children, mainly between ages $5-16$ is a serious matter of concern. Once a child step to the age of five, they are considered more or less safe from nutritional disorders. Nevertheless, little attention is paid to the quality of life. School going children are hardly thought of, as "at risk" population, but this phase is a unique intervention point in the life cycle [5].

Our pre-existing idea about education is that better educated parents are more successful in protecting or improving their children's health status, but this study explores that parental education does not play a key factor that were significantly associated with underweight and obesity status among children aged between 5-16 years. Analysis showed that underweight or obesity percentage does not differ significantly among the students with the advancement of parental education.

Again, another vital consideration for child nourishment is an occupation of parents. It is customarily believed that the father is the primary earner and decision maker of a family and so their higher level of education and occupational status plays an important role to ensure better nutritional status of children. This study explains that irrespective of male and female students, type of parental occupation does not have much effect on their child's health.

It is well recognized and very common concept for the Indians that sharing of foods in a family member may act as a protective factor and affects their child nutrition and leads to underweight of their children. But the present study reveals that the degree of association between number of family members and child nutrition is not statistically significant. 
As this study reveals that, there is no significant impact of parental education, type of parental occupation and number of family members on children's nutritional health, it can be concluded that lack of understanding about regular exercise and the benefits of doing so among the parents and students could be a reason for such scenario. We can also presume that specific health education is needed for both parents and students, otherwise we cannot prevent under nutrition as well as obesity. Because it is revealed from this study that neither obesity nor underweight is related to vital indices like education, type of occupation and number of household members. It is imperative to note that despite this caveat, the findings of this study should be useful for identifying classes of children, particularly who are at high risk of underweight and obesity. [1] The findings also imply that the prevalence of underweight and obesity can be reduced by 'proper health awareness', by introducing 'specific health education' in the school curriculum, by improving the educational levels of parents particularly mothers, by developing knowledge of parents about 'child health'. Such undertaking should be combined with programmes that raise women's awareness of the nutritional requirement of the children. [1] It is also a scientific fact that proper nutrition can not isolate without physical activity; regular exercise or participation in sports and games of the children and adolescents is mandatory for growth and development and overall well being.

\section{Acknowledgement}

We sincerely express our gratitude to Sarbashiksha Mission, Bardhaman for such initiatives. We are thankful to School administrators, teachers and students for participating in this study. We also thankful to our colleagues, friends for their consistent support.

\section{References}

[1] Child Nutrition in India: Vinod K. Mishra, Subrata Lahiri, and Norman Y. Luther. National Family Health Survey Subject Reports Number 14. June 1999.

[2] UNICEF REPORT 2006.

[3] $47 \%$ of children in India are underweight: UNICEF; Info Change News \& Features, May 2006.

[4] Magnitude of Malnutrition and Iron Deficiency Anemia among Rural School Children: An Appraisal: Rachana Bhoite, Uma Iyer ,Foods and Nutrition Department, M.S. University of Baroda, Vadodara.; ASIAN J. EXP. BIOL. SCI. VOL 2 (2) 2011: 354-361.

[5] Kotecha, P.V. (2008) Micronutrient malnutrition in India: Let us say "no" to it. Indian J community Med; 33: 9-10.

[6] Mason, J.B (2003) At least one third of poor countries diseases burden is due to malnutrition: diseases control priorities project: working paper no. 1, March 2003.

[7] VV Khadilkar, AV Khadilkar, AB Borade and SA Chiplonkar: Body Mass Index Cut-offs for Screening for Childhood Overweight and Obesity in Indian Children, INDIAN PEDIATRICS, VOLUME 49, JANUARY 16, 2012.

[8] Kapil U, Singh P, Pathak P, Dwivedi SN, Bhasin S. Prevalence of obesity among affluent adolescent school children in Delhi. Indian Pediatr. 2002; 39: 365-8.

[9] Emmanuel Skoufias: Parental Education and Child Nutrition in Indonesia International Food Policy Research Institute, Washington DC, Bulletin of Indonesian Economic Studies Vol 35 No 1, April 1999, pp. 99-119.

[10] Socioeconomic Status And Child Development: Robert H. Bradley and Robert F. Corwyn, Center for Applied Studies in Education, University of Arkansas at Little Rock, 2801 S. University Ave., Little Rock, Arkansas 72204.

[11] The Dual Burden of Overweight and Underweight in Developing Countries: Marya Khan, research associate with the Population Reference Bureau; Published at Population Reference Bureau, March 2006.

[12] Is Frequency of Shared Family Meals Related to the Nutritional Health of Children and Adolescents? Amber J. Hammons, PhD* and Barbara H. Fiese, PhD; Published at American Academy of Pediatrics on $2^{\text {nd }}$ May, 2011.

[13] WHO, information series on school health, document 13, Malaria prevention and control: An important responsibility of a health promoting school, 2007.

[14] The influence of parental income on children outcomes: Susan E. Mayer, Associate Professor in the Harris School of Public Policy Studies at the University of Chicago; was published by Knowledge Management Group, Ministry of Social Development, Te Manatu Whakahiato Ora 2002. 